\section{Acute Necrotising Encephalopathy of Childhood Secondary to Rotaviral Diarrhoea}

A one-year-old male child presented with complaints of fever, vomiting and loose stools for 4 days. After 6 days, symptoms subsided but child developed rapid worsening sensorium and hepatomegaly. Investigations revealed marked elevation in serum transaminases (aspartate aminotransferase (AST) 10491 IU/L, alanine aminotransferase (ALT) (8990 IU/L), serum albumin was $2.4 \mathrm{gm} / \mathrm{dL}$, prothrombin time, 32.5 seconds (International normalized ratio (INR) was 2.77, which improved to 1.53 after vitamin K supplements. Serum ammonia was initially $136.7 \mu \mathrm{g} / \mathrm{dL}$ which improved to $23 \mu \mathrm{g} / \mathrm{dL}$ on treatment. Cerebrospinal fluid analysis (CSF) was normal. Dengue NS1, IgM and IgG, HBsAg, hepatitis C IgG, hepatitis A $\operatorname{IgM}$, hepatitis E IgM and ELISA for HIV were negative. Stool rotavirus antigen was positive.

Magnetic resonance imaging (MRI) of brain showed hyperintensities in the bilateral caudate nuclei, putamen, globus pallidus and restricted diffusion in the bilateral basal ganglia suggestive of acute necrotizing encephalopathy. Child was treated with intravenous methyl prednisolone $(30 \mathrm{mg} / \mathrm{kg} /$ day $)$ for 3 days, followed by oral prednisolone at $2 \mathrm{mg} / \mathrm{kg} /$ day for 15 days, which was gradual tapered over next one month. On day 6 of hospitalisation, the child's sensorium improved and he was discharged with feeding tube in situ, with residual neurological deficit, and AST of $81 \mathrm{IU} / \mathrm{L}$ and ALT of $1250 \mathrm{IU} / \mathrm{L}$. On followup after 45 days of illness, his liver function tests have normalized, he can feed without the feeding tube, can speak monosyllables and can recognize parents.

Acute necrotizing encephalopathy is a para-infectious disease triggered by viral infections, most commonly by influenza and HHV 6 [1-3]. The most likely hypothesis for the pathogenesis of ANE is the exaggerated inflammatory response to viral infection leading to liver dysfunction, acute renal failure, shock, and disseminated intravascular coagulation. In nervous system, the permeability of vessels is altered without vessel wall disruption [3].

Our patient had a history of viral gastroenteritis and the stool rotavirus antigen was positive. Neurological manifestations associated with rotavirus have been described [4]. Thus, we consider rota virus as the possible etiology for ANE. Definitive treatment guidelines for ANE have not been formulated but antiviral therapy, immunomodulatory treatment, antithrombin III, therapeutic hypothermia and cyclosporin A have been variably used [1,3]. ANE is associated with a high mortality and less than $10 \%$ of patients recover completely [3]. In conclusion, a high index of suspicion for ANE is needed in a previously healthy child with sudden onset neurological symptoms following acute febrile illness.

Vishrutha SuJITh Poojari, Ira ShaH aNd NAMAN S SHETTY* Pediatric Gastroenterology, Hepatology and Nutrition Department, BJ Wadia Hospital for Children, Mumbai, Maharashtra, India.

\section{REFERENCES}

*naman.shetty@hotmail.com

1. Yoganathan S, Sudhakar SV, James EJ, Thomas MM. Acute necrotising encephalopathy in a child with H1N1 influenza infection: A clinicoradiological diagnosis and follow-up. BMJ Case Rep. 2016; 2016:ber 2015213429.

2. Kulkarni R, Kinikar A. Encephalitis in a child with H1N1 infection: First case report from India. J Pediatr Neurosci. 2010;5:157-9.

3. Wu X, Wu W, Pan W, Wu L, Liu K, Zhang HL. Acute necrotizing encephalopathy an under recognized clinicoradiologic disorder. Mediators Inflamm. 2015;2015:79257.

4. Shiihara T, Watanabe M, Honma A, et al. Rotavirus associated acute encephalitis/encephalopathy and concurrent cerebellitis: report of two cases. Brain Dev. 2007;29:670-73.

\section{The Neonatal Resuscitation Protocol: Keep It Simple?}

Neonatal intensive care practice has its moments I would say; several actually. Sending a micropreemie home, watching a meconium aspiration pneumonia improve on high frequency ventilation and nitric oxide, managing to insert a life-saving central line catheter into a fine thread like vein, cherishing the normal neurodevelopment of a critically ill infant; all of these and more make the effort worth it. Why then, did the neonatologist in me decide to put myself at risk of criticism with this manuscript which could ripple some still waters? Albeit I am no virtuoso in the field of medical research, having faced the maelstrom of intensive care on my feet for over 10 years, I wish to comment on a guideline that makes a difference to every day practice. The neonatal resuscitation protocol (NRP) for the term newborn, can be described as "daily bread" to the genus of intensivists called neonatologists and has undergone several modifications over the last decades. Thyself followed, with great fervour, the 'reforms' made to the protocol [1]. Over the ensuing paragraphs I intend to raise my reservations on the tipping balance in NRP, between the quest for evidence based practices and pragmatism. 


\section{CORRESPONDENCE}

Those who work with older children and adults know that their patients crash mainly due to cardiac reasons. Basic life support training hence focusses on pushing hard and fast to get the circulation going [2]. On the other hand, babies who do not seem fine at birth are typically so because of prior hypoxemia and all the problems that result: hence, the focus on 'breathing'. Either stimulate him (forgive the literal gender bias) to cry, or drive air in by positive pressure ventilation, and most of the other problems (bradycardia, poor tone) sort themselves out. There is a small proportion who need chest compressions and drugs, though [3]. Experiments on animals, and results from various clinical studies, apparently guided changes in the protocol and aimed at improving outcomes, which is indubitably the correct way forward.

Did we outbid practicality in this quagmire of trying to add more and more boxes and branches to the resuscitation algorithm? Ab initio, everything seemed undeniably robust. Certain vital information was essential at birth (the first 'box' in the algorithm), and all it took to decide if the baby was fine was that he should cry with gusto and look all flexed and pink within minutes. But the contents of these so-called boxes kept changing, with its members moving in and out with every update. Even if you manage to let that pass, some of the additions that came in later further along the algorithm does want to make you sit up and roll your eyes. It sounded reasonable to attach a pulse oximeter probe for those who needed more supports; after all, we swear by primum nonnocere and oxygen does do harm [4]. But sticking ECG leads as time ticks on? And then staring at the monitor for precious seconds to get readings and act: now that is a tough one to comprehend in the chaos. Specifically when the evidence is tenuous at best; as slippery as the baby in fact [5]. Whatever happened to years of training listening to the lub-dub through the instrument we wield as the mark of a doctor?

Let that go by too. What seemed completely overboard and actually made me gape incredulously was this. Suppose the little fellow needs chest compressions too (remember someone is already ventilating him from the head end by then); the 'compressor' who was on one side of the baby needs to move to the head end and nudge the 'ventilator' to swap positions. The picture that comes to mind is that of an entangled crochet of the operators' forearms and hands. Why not continue the twothumb chest compression from one side, allowing the airway person to continue his good work from the head end (especially considering the stability of the more important airway)? Seemingly to make way for a third person to insert the umbilical vein canula, if required. What happened to the other side of the baby, usually the left? Try telling me that clinical examination and procedures need to be done from the right side of the patient (one of the first 'rules' drilled into a medical student). The foundation for this custom is quite simply convention [6]. A right hander may do a better job while examining asymmetric organs like the liver, spleen or heart; but the umbilical stump? Try it on a mannequin, makes no difference at all- right or not right!
I could go on and on. Adding to the angst is the concern that a student/ trainee is reprimanded; or worse-still, failed in the objective structured clinical examination stations, for not strictly adhering to the rule-book. Not to mention the ever looming medico-legal issues that can be pursued by those who go to court for 'errors' during resuscitation.

At the end of the road, all we need to do most of the time, is to reverse the hypoxemia and hypercarbia by effectively ventilating the lung, and the heart and brain follow suit. But no, we like to complicate everything. I am reminded of the historical Rube Goldberg machine!

On a more serious note, if we set aside a miniscule fortunate fraction of the population, our country's less privileged interiors are still grappling with bare minimum availability of equipment and trained personnel $[7,8]$. With great efforts, the neonatal mortality has dropped from 38 to 23.5 per 1000 live births [9]. But we have miles to go. A close look at the vast differences in statistics within the country seems to indicate the need for very specific regional and local microplanning. In such conditions, we need to earnestly contemplate the practical applicability of the ever evolving NRP; and consider local, regional logistics and readiness before blanket recommendations are made. An additional section in the NRP guidelines on adoption of new guidelines at various levels of healthcare may be added to address similar issues.

\section{REFERENCES}

Femitha Pournami Department of Neonatology, Kerala Institute of Medical Sciences, Trivandrum, India. femi_shifas@yahoo.com

1. Neonatal resuscitation: 2020 American Heart Association guidelines for Cardio- pulmonary resuscitation and Emergency Cardiovascular care. Circulation 2020: 142:S524-50.

2. Part 1: Executive Summary. 2020 American Heart Association guidelines for Cardio- Pulmonary Resuscitation and Emergency Cardiovascular Care. Circulation 2020:142:S337-57.

3. Guidelines on basic neonatal resuscitation. World Health Organisation 2012. Accessed January 12 January 2021.Available from: https://apps.who.int/iris/bitstream/handle/ 10665/75157/ 9789241503693eng.pdf;jsessionid $=$ CF1BCA6B69AF844E2831A0606 FDE0419? sequence $=1$.

4. Perrone S, Bracciali C, Di Virgilio N, Buonocore G. Oxygen use in neonatal care: A two-edged sword. Front Pediatr. 2017;4:143.

5. Johnson PA, Schmölzer GM. Heart rate assessment during neonatal resuscitation. Healthcare (Basel). 2020;8:43.

6. Qayyum MA, Sabri AA, Aslam F. Medical aspects taken for granted. Mcgill J Med. 2007;10:47-49.

7. Das MK, Chaudhary C, Mohapatra SC, et al. Improvements in essential newborn care and newborn resuscitation services following a capacity building and quality improvement program in three districts of Uttar Pradesh, India. Indian J Com Med. 2018;43:90-6.

8. Thakre R. Neonatal resuscutation guidelines: India specific concerns. Indian Pediatr. 2017;54:333.

9. Kumar P, Singhal N. Mapping Neonatal and Under 5 mortality in India. Lancet. 2020;395:1591-93. 